\title{
Circadian gene PER1 senses progesterone signal during human endometrial decidualization
}

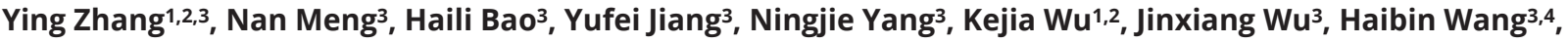 \\ Shuangbo Kong3,4 and Yuanzhen Zhang1,2 \\ 1Department of Obstetrics and Gynecology, Zhongnan Hospital of Wuhan University, Wuhan, Hubei, China \\ ${ }^{2}$ Hubei Clinical Research Center for Prenatal Diagnosis and Birth Health, Wuhan, Hubei, China \\ ${ }^{3}$ Fujian Provincial Key Laboratory of Reproductive Health Research, School of Medicine, Xiamen University, Xiamen, Fujian, China \\ ${ }^{4}$ Reproductive Medical Center, The First Affiliated Hospital of Xiamen University, Xiamen, Fujian, China
}

Correspondence should be addressed to Y Zhang or S Kong or H Wang: zhangyuanzhen@vip.sina.com or shuangbo_kong@163.com or haibin.wang@vip.163.com

\begin{abstract}
Progesterone is an important hormone for female reproduction; however, how the fluctuation of progesterone acts upon reproductive processes remains largely unknown. Mounting evidence indicates a pivotal role of the circadian clock in sensing hormone dynamics for homeostatic regulation of physiological functions. Therefore, we sought to determine whether clock genes respond to progesterone signaling in female reproductive system. In this study, we tested the hypothesis that the circadian system could respond to progesterone signaling during human endometrial decidual transformation. The expression of the circadian gene PER1 increased immediately and remained elevated during human endometrial decidualization. The progesterone receptor activated PER 1 transcription by directly binding to its promoter from the onset of the stromal proliferation-differentiation transition. PER1 knockout significantly downregulated the expression of some PGR target genes, and attenuated human endometrial decidual transformation by expediting FOXO1 protein degradation. In conclusion, progesterone could control the female reproductive process through sustained feedback from the circadian gene PER1, which is probably involved to $\mathrm{P}_{4}-\mathrm{PR}$ signaling responsiveness in the initiation and maintenance of decidualization.
\end{abstract}

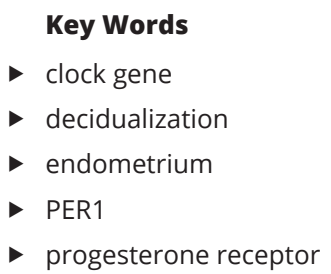

Journal of Endocrinology (2019) 243, 229-242

\section{Introduction}

Progesterone $\left(\mathrm{P}_{4}\right)$ is essential for regulating mammalian female reproduction. In the human menstrual cycle, $\mathrm{P}_{4}$ secretion is minimal during the follicular phase and increases after ovulation, which plays a major role in preparing the uterus for embryo implantation and favors the establishment and maintenance of pregnancy. The effects of $\mathrm{P}_{4}$ are mediated by $\mathrm{P}_{4}$ binding with its cognate nuclear receptors, progesterone receptors (PRs, including PRA and PRB; both are encoded by the PGR gene), and the pleiotropic action of $\mathrm{P}_{4}$ mainly relies on PR signaling that occurs in a cell type-specific manner. Endometrial stromal cells, which are principal targets for $\mathrm{P}_{4}$ in the uterus, undergo a dynamic morphological transformation process involving a proliferation-differentiation transition that is defined as decidualization (Gellersen \& Brosens 2014) and is sustained by elevated $\mathrm{P}_{4}$ levels in the luteal phase (Gellersen \& Brosens 2003). It has been confirmed that compromised decidualization associated with aberrant $\mathrm{P}_{4}$-PR signaling could lead to a variety of infertility diseases, such as endometriosis (EMS). 
However, our understanding of how $\mathrm{P}_{4}$ modulates this process is still far from sufficient.

The circadian clock system exists to adapt to environmental signals such as hormonal dynamics, lightdark cycles, feeding and fasting, chronothermal signals or sleep-wake cycles (Papazyan et al. 2016). The rhythm pacemaker is generated within the suprachiasmatic nuclei (SCNs) of the hypothalamus and operates in both central and peripheral tissues, including the uterus (Mohawk et al. 2012, Miller \& Takahashi 2013). The canonical circadian system consists of four genes (and their analogs): circadian locomotor output cycles kaput (CLOCK), brain muscle arnt-like 1 (BMAL1), period (PER1, PER2, and $P E R 3)$ and cryptochrome (CRY1 and CRY2). It has been validated that all these genes are expressed in the human, rat and mouse uterus, and some of them can be altered by ovarian hormones (Johnson et al. 2002, Nakamura et al. 2005, 2008, 2010, He et al. 2007, Hirata et al. 2009). PER2 deficiency leads to flattened diurnal oscillation of all the core clock genes and to disorganized decidual responses in human endometrial stromal cells (HESCs) after continuous decidualization for 4 days by blocking the cell cycle, but whether clock genes mediate the responses of HESCs to $\mathrm{P}_{4}$ signals remains unclear (Muter et al. 2015). In addition, knockout of Pgr results in decreased expression of many rhythm genes in the mouse uterus, e.g., Per1 (Rubel et al. 2012); thus, we hypothesized that the regulatory mechanism controlling decidualization responses upon $\mathrm{P}_{4}$ signaling might be mediated by other clock genes, such as PER1, rather than PER2.

To test this hypothesis, we investigated whether the expression of these clock genes changed at the beginning of the differentiation transition, a question that to our knowledge has not been addressed by previous studies. The conventional concept holds that the process of stromal cell differentiation commences with elevated intracellular cAMP levels and is then sustained by rising $\mathrm{P}_{4}$ levels (Gellersen \& Brosens 2003, 2014). cAMP signaling is also an intrinsic part of the pacemaker pathway for SCN cells and most peripheral cells throughout the body (Yagita \& Okamura 2000, O'Neill et al. 2008, Zmrzljak et al. 2013). Our data show that the immediate PER1 response to $\mathrm{P}_{4}$ signaling, but not to cAMP signaling, at the early stage of decidualization. To explore the regulatory effects of $\mathrm{P}_{4}$ on clock genes, we used a CRISPR/Cas9 approach and a luciferase reporting system, through which we verified that the transcriptional activation of PER1, the core circadian gene, is directly regulated by PR beginning at the initiation of decidualization (Large \& DeMayo 2012, Patel et al. 2015). Remarkably, PER1 deficiency leads to declined progesterone signal responsiveness and compromised decidualization at least partly by decreasing forkhead box O1 (FOXO1) protein stability.

\section{Materials and methods}

\section{Patient selection and ethical approval}

The study was performed based on the approval of the Medical Ethics Committee of Zhongnan Hospital of Wuhan University (2017061). Fifty-five women (age range 23-42 years) were recruited from the Reproductive Medicine Center of Zhongnan Hospital from January to December (2018). Written informed consent was obtained from all participants according to the guidelines of the Declaration of Helsinki. For analysis of the expression of PER1 mRNA levels, a total of 46 biopsies were obtained from patients who had regular ovulatory menstrual cycles. According to the patients' cycle day and urinary luteinizing hormone (LH) surges, collection of proliferative and luteal phase samples was timed. Additionally, nine fresh biopsies of endometria in the proliferative phase were processed for primary cultures. All subjects were treated without any hormones for at least 3 months before testing.

\section{Primary cell culture, transfection, siRNA-mediated PER1 silencing and decidualization treatments}

Samples were isolated and cultured as described previously (Muter et al. 2015). According to the manufacturer's instructions, RNA interference experiments were carried out, generally with $10 \mathrm{mM}$ siRNA and Lipofectamine RNAiMAX (Invitrogen). Twenty-four hours after transfection, primary HESCs were decidualized in DMEM/F-12 containing 2\% charcoal-stripped FBS (CS-FBS, Biological Industries, HaZafon, Israel), $0.5 \mathrm{mM}$ dibutyryl cAMP (db-cAMP, MCE, Princeton, USA) (a cAMP analog) and $1 \mu \mathrm{M}$ medroxyprogesterone 17-acetate (MPA, Sigma) (a progesterone analog), and cells were collected for up to 6 days for RNA or protein extraction with medium replacement every $48 \mathrm{~h}$. The sequence of the siRNA is listed in Table 1. Scramble siRNA was used as a negative control. All experiments were carried out before the fifth cell passage. The volumes of fifty cells in each group were determined by using ImageJ software.

\section{Immortalized HESC culture and decidualization treatments}

An immortalized HESC line was purchased from the American Type Culture Collection (ATCC® CRL-4003 ${ }^{\mathrm{TM}}$ ) 
Table 1 Sequences used in this study.

\begin{tabular}{l}
\hline Gene \\
\hline SiRNA sequence \\
PER1 \\
CRISPR/Cas9 sgRNA \\
PER1 \\
PGR \\
ChIP-PCR primer \\
PER1-PRE-1 \\
PER1-PRE-2 \\
PER1-PRE-3 \\
PER1-PRE-4 \\
Overexpression vector primer \\
FOXO1 \\
qPCR primer \\
GAPDH \\
PER1 \\
PER2 \\
PER3 \\
CRY1 \\
CRY2 \\
CLOCK \\
BMAL1 \\
PRL \\
IGFBP1 \\
FOXO1 \\
PGR \\
DKK1 \\
THBS1
\end{tabular}

\begin{tabular}{l}
\hline Forward primer \\
GTGGGAGTCTTCTATGGTT \\
GGCCACGCTGCAGTACGCAC \\
AAGACGAAATTACGACGGC \\
CTCTGTTGTATGACTTGTGACT \\
GGCTCACGCCTGTAATCTTAT \\
TGTACAAAGTGCGCCTTA \\
TGGCCTGCTGACCAATGA \\
GGCCACTAGTATGGATTACAAGGATGACGACGATAA \\
GATGGCCGAGGCGCCTCAGGT \\
ACGGATTTGGTCGTATTGGG \\
TCAAGTGGCAATGAGTCCAA \\
TGAATGGATACGCGGAATTT \\
CCTGTCTGTCCTCTGTTGTC \\
TTGCTTGATGCAGATTGGAG \\
GCCTGGATAAGCACTTGGAA \\
AGCATGGTCCAGATTCCATC \\
GCCATCTCGATTATGTTCTG \\
CTACATCCATAACCTCTCCTCAG \\
AGAGTCGTAGAGAGTTTAGC \\
GGCAGCCAGGCATCTCAT \\
TGTATTTGTGCGTGTGGGTG \\
TTCCAACGCTATCAAGAACCTGC \\
CTGTGCGGGCAGAAAAGGG \\
\hline
\end{tabular}

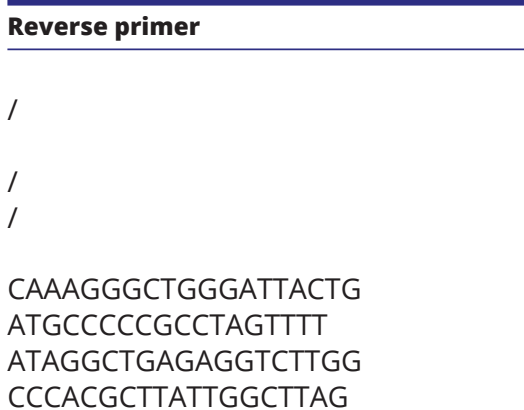

ATGGATCCTCAGCCTGACACCCAGCTAT

CGCTCCTGGAAGATGGTGAT
TGTAGGCAATGGAACTGCTG
AGCTCCTTCCCACTGTCGT
GCTGCTTCTCGAAGTAATGA
GGGAAGCCTCTTAGGACAGG
GTCCCACAGGCGGTAGTAGA
ATGACTGCCCCACAAGCTAC
CTCATTGTCTGGTTCGTTGT
GGGCTTGCTCCTTGTCTTC
ACACTGTCTGCTGTGATAA
TGGGTCAGGCGGTTCATAC
TACAGCCCATTCCCAGGAAG
TGCCGCACTCCTCGTCCTCT
TTGGACACCACGCTGAAGACC

$B M A L 1$, aryl hydrocarbon receptor nuclear translocator like; CLOCK, clock circadian regulator; CRY1, cryptochrome circadian regulator 1; CRY2, cryptochrome circadian regulator 2; DKK1, dickkopf WNT signaling pathway inhibitor 1; FOXO1, forkhead box O1; GAPDH, glyceraldehyde-3-phosphate dehydrogenase; IGFBP1, insulin-like growth factor-binding protein 1; PER1, period 1; PER2, period 2; PER3, period 3; PGR, progesterone receptor; PRE, progesterone response element; PRL, prolactin; THBS1, thrombospondin 1.

and cultured as described previously (Liao et al. 2015). To synchronize the circadian transcriptome (Reddy et al. 2007), immortalized HESCs were treated with $100 \mathrm{nM}$ dexamethasone (Sigma-Aldrich) for $30 \mathrm{~min}$, then treated with $10 \mathrm{nM}$ estradiol to represent an undifferentiated stromal cell state or with $0.5 \mathrm{mM}$ db-cAMP plus $1 \mu \mathrm{M}$ MPA to induce decidualization, respectively. The cells were cultured for up to 8 days for RNA or protein extraction with medium replacement every $48 \mathrm{~h}$. All experiments were carried out in ten passage of the stromal cells.

\section{Knockout of the PER1 and PGR genes with the CRISPR/Cas9 system}

The sgRNAs target PER1 gene (NC_000017.11) and PGR gene (NC_000011.10) were designed on the website (https://zlab.bio/guide-design-resources). Each $20 \mathrm{bp}$ target sequence was subcloned into the pL-CRISPR.EFS. GFP vector (Addgene plasmid \#57818) to construct sgRNA expression vectors. The CRISPR/Cas9 target sequences are listed in Table 1. HEK293T cells were plated in $15 \mathrm{~cm}$ culture dishes 1 day before transfection. When the cells reached $80 \%$ confluence, they were transfected with the lentivirus packaging plasmid psPAX2 (Addgene plasmid \#12260), pMD2.G (Addgene plasmid \#12259), and the sgRNA plasmids with Lipofectamine ${ }^{\mathrm{TM}} 3000$ reagent (Invitrogen) according to the manufacturer's instructions. Seventy-two hours after transfection, the virus was concentrated by ultracentrifugation and then used to infect immortalized uterine stromal cells. Seventy-two hours after infection, these cells were trypsinized and single fluorescence-positive cell was selected for propagation. After several days of culturing, the genomic DNA in the clone derived from single fluorescence-positive cell was extracted for amplifying the sequences that crossing the splice sites with a high-fidelity enzyme (Toyobo, Osaka, Japan), and the PCR products were ligated into the pEASY-T cloning vector (Transgen, Beijing, China) for Sanger sequencing to confirm the knockout of the target genes. Clones with frameshift mutations in both alleles were chosen for the further experiments.
(C) 2019 Society for Endocrinology Published by Bioscientifica Ltd. Printed in Great Britain 


\section{ChIP-PCR}

Formaldehyde (1\%) was used to fixed HESCs in $10 \mathrm{~cm}$ culture dishes for $10 \mathrm{~min}$ at room temperature, and then the fixation was stopped with $125 \mathrm{mM}$ glycine on ice for 10 min. After Dulbecco's phosphate-buffered saline (DPBS) washes, the cells were scraped and centrifuged for $5 \mathrm{~min}$ at $700 \mathrm{~g}$ and $4^{\circ} \mathrm{C}$. The sediment was resuspended in $1 \mathrm{~mL}$ of lysis buffer 1 (0.25\% Triton X-100, 0.5\% NP-40, 10\% glycerol, $1 \mathrm{mM}$ EDTA, $50 \mathrm{mM}$ HEPES and $140 \mathrm{mM} \mathrm{NaCl}(\mathrm{pH}$ 7.55)) and rotated for $10 \mathrm{~min}$ before centrifugation for $5 \mathrm{~min}$ at $1400 \mathrm{~g}$ and $4^{\circ} \mathrm{C}$. The sediment was then resuspended in $1 \mathrm{ml}$ of lysis buffer 2 ( $1 \mathrm{mM}$ EDTA, $0.5 \mathrm{mM}$ EGTA, $200 \mathrm{mM}$ $\mathrm{NaCl}$, and $10 \mathrm{mM}$ Tris- $\mathrm{HCl}(\mathrm{pH} 8.0)$ ) and rotated for $10 \mathrm{~min}$ at room temperature before centrifugation for $5 \mathrm{~min}$ at $1400 \mathrm{~g}$ and $4^{\circ} \mathrm{C}$. The sediment was resuspended in $600 \mu \mathrm{L}$ of lysis buffer 3 (0.5\% N-lauroylsarcosine, $0.1 \%$ sodium deoxycholate, $1 \mathrm{mM}$ EDTA, $0.5 \mathrm{mM}$ EGTA, $100 \mathrm{mM} \mathrm{NaCl}$, and $10 \mathrm{mM}$ Tris- $\mathrm{HCl}$ (pH 8.0)) and sonicated for $10 \mathrm{~min}$ at $4^{\circ} \mathrm{C}$ in a Bioruptor ${ }^{\circledR}$ Pico sonication device (Diagenode, Liège, Belgium). The buffers were supplemented with protease and phosphatase inhibitor cocktails (Roche). Later, $60 \mu \mathrm{L}$ of $0.25 \%$ Triton $\mathrm{X}-100$ were added and the mixture was centrifuged for $10 \mathrm{~min}$ at $20,000 \mathrm{~g}$ and $4^{\circ} \mathrm{C}$, the supernatant was then complexed overnight at $4^{\circ} \mathrm{C}$ with a progesterone receptor antibody (Cell Signaling Technology) bound to Protein A Dynabeads. Polyclonal anti-rabbit IgG was used as a negative control. After complexing, the samples were eluted successively at room temperature with the following buffers: low-salt buffer $(1 \%$ Triton X-100, 0.5\% sodium deoxycholate, $2 \mathrm{mM}$ EDTA, $50 \mathrm{mM}$ HEPES, and $150 \mathrm{mM} \mathrm{NaCl})$; high-salt buffer $(1 \%$ Triton X-100, 0.5\% sodium deoxycholate, $2 \mathrm{mM}$ EDTA, $50 \mathrm{mM}$ HEPES, and $500 \mathrm{mM} \mathrm{NaCl}) ; \mathrm{LiCl}$ buffer $(0.5 \%$ NP-40, 0.5\% sodium deoxycholate, $1 \mathrm{mM}$ EDTA, $250 \mathrm{mM}$ $\mathrm{LiCl}$, and $10 \mathrm{mM}$ Tris-HCl (pH 8.0)); and Tris-EDTA buffer (10 mM Tris-HCl ( $\mathrm{pH} 8.0$ ) and $1 \mathrm{mM}$ EDTA). Then, the chromatin was eluted with $200 \mu \mathrm{L}$ of elution buffer $(1 \%$ SDS and $10 \mathrm{mM} \mathrm{NaHCO}$ ). After an overnight incubation at $65^{\circ} \mathrm{C}$ to reverse-crosslink the proteins and the DNA, $0.2 \mathrm{mg} / \mathrm{mL}$ protease $\mathrm{K}$ was added for incubation at $37^{\circ} \mathrm{C}$, and before DNA purification, the sample was incubated for $1 \mathrm{~h}$ at $55^{\circ} \mathrm{C}$. The purified DNA was amplified by PCR, and the primer sequences are listed in Table 1.

\section{F0X01 overexpression plasmid construction}

The human FOXO1 primer sequences for PCR are listed in Table 1. The coding sequence of the FOXO1 gene (NC_000013.11) was amplified by PCR from the cDNA of

(c) 2019 Society for Endocrinology Published by Bioscientifica Ltd. Printed in Great Britain immortalized HESCs, and the PCR product was inserted between the SpeI and BamHI sites of the pLVX-IRES-ZsGreen vector. The FOXO1 overexpression plasmid was extracted with a HiPure Plasmid EF Maxi Kit (Magen, Guangzhou, China). Sequencing and comparison with GenBank sequences were performed. HEK293T cells were plated in $15 \mathrm{~cm}$ culture dishes 1 day before transfection. When the cells reached $80 \%$ confluence, they were transfected by the lentivirus packaging plasmid psPAX2, pMD2.G, and the FOXO1 overexpression plasmid with Lipofectamine ${ }^{\mathrm{TM}}$ 3000 reagent according to the manufacturer's instructions. Seventy-two hours after transfection, the virus was concentrated by ultracentrifugation and then used to infect primary HESCs for $24 \mathrm{~h}$. Primary HESCs were then transfected with siPER1 for $24 \mathrm{~h}$, and then treated with cAMP plus MPA up to 4 days.

\section{Dual-luciferase reporter assay}

The assay was performed using two reporter vectors containing the firefly luciferase vector pGL3-Basic and the Renilla luciferase vector pRL-TK (Promega). Four PER1 promoter regions were respectively amplified by PCR from human genomic DNA and were subcloned in the upstream of the luciferase gene of pGL3-Basic vector. HEK293T cells were plated into 24 -well plates, each plate was transiently co-transfected by these two vectors with or without a PR overexpression vector (pCMV-HA-PR) (Xin et al. 2018) and MPA using the Lipofectamine ${ }^{\mathrm{TM}} 3000$ reagent. Thirtysix hours after transfection, cell lysates were harvested, and then luciferase activities were measured by the DualLuciferase Reporter System (Promega) according to the manufacturer's instructions. The firefly luciferase activities were normalized to the activities of Renilla luciferase.

\section{RNA isolation and qRT-PCR}

Total RNA was extracted using RNAiso Plus (TaKaRa) according to the manufacturer's protocol. Total RNA $(1 \mu \mathrm{g})$ from each sample was treated with DNase I and reverse transcribed using the PrimeScript ${ }^{\mathrm{TM}} \mathrm{RT}$ reagent Kit (TaKaRa). Real-time PCR assay was performed using TB Green ${ }^{\circledR}$ Premix Ex Taq ${ }^{\mathrm{TM}}$ II (TaKaRa) with the QuantStudio 5 Real-Time PCR System (Applied Biosystems). The analysis of amplification curve and melting curve were performed to determine the optimal primer concentration and specificity of amplifications. The primer sequences used are listed in Table 1. The analysis of standard curve was performed for estimating the efficiency of the amplifications and ranges of the assay. Real-time PCR 
assay both with mRNA template and without cDNA template were tested as negative control. The variances of cDNA was normalized against the levels of the Homo sapiens GAPDH housekeeping gene. The expression levels of the samples were calculated using the $\Delta \Delta \mathrm{Ct}$ method. All measurements were performed at least in triplicate, the average of the replication was used for statistical analysis.

\section{Immunofluorescence staining}

HESCs were fixed with absolute methanol at room temperature for $10 \mathrm{~min}$. After three washes with PBS, the cells were permeabilized with $0.2 \%$ Triton X-100 in $1 \times$ PBS for $15 \mathrm{~min}$ and blocked with $0.5 \%(\mathrm{w} / \mathrm{v})$ bovine serum albumin (BSA, Sigma-Aldrich) for $1 \mathrm{~h}$ at room temperature. Anti-PER1 (Abcam), FOXO1 (Cell Signaling Technology), Vimentin (Epitomics, Burlingame, USA), and cytokeratin (DAKO) primary antibodies were used, and then the cells were incubated with fluorescence (cyanine 3)-conjugated secondary antibodies to visualize the signals with DAPIstained nuclei $(1 \mu \mathrm{g} / \mathrm{mL}$, Sigma-Aldrich). Full descriptions of the antibodies, including catalog numbers and concentrations, can be found in Table 2 . The cells without primary antibody served as negative control.

\section{Western blot analysis}

Protein extraction and Western blot analysis were performed as described previously (Zhang et al. 2014). The antibodies used are listed in Table 2 . The intensity of bands was determined by Image Lab software, and quantitative analysis of the grayscale value of each target protein was normalized to GAPDH. The gel calculation was based on three different replications, and the average grayscale value of the replication was used for statistical analysis. Only one representative film was shown in our manuscript.

\section{Protein degradation assay}

To determine the half-life of FOXO1 protein, PER1 knockout and control (empty vector) immortalized HESCs with $30-40 \%$ confluency were seeded in $3.5 \mathrm{~cm}$ culture dishes a day before induction of decidualization for 2 days followed by treatment with cycloheximide (CHX, EMD Millipore), a protein synthesis inhibitor, for 0 , 1, 2 or $4 \mathrm{~h}$. The same treatments were implemented with the calpain inhibitor MG101 (Selleck), the proteasome inhibitor MG132 (Selleck), and the autophagy inhibitor 3-methyladenine (3-MA, Selleck). A group treated with DMSO served as the negative control group.

\section{Statistical analysis}

These data are derived from entirely separate samples and are presented as means \pm S.E.M. (standard error of the mean). $n$ represents the number of independent experiments. Statistical analysis was performed using GraphPad Prism 8 Software and SPSS 17.0 Software. Analysis was performed with Student's unpaired two-tailed $t$-test, one-way ANOVA with Bonferroni's post hoc test or two-way repeatedmeasures ANOVA with Bonferroni's post hoc test. A level of $P<0.05$ was taken to indicate statistical significance.

\section{Results}

\section{Circadian gene PER1, rather than other clock genes, senses decidual signals}

We measured the expression levels of seven core clock genes every $4 \mathrm{~h}$ for $32 \mathrm{~h}$ in immortalized HESCs in vitro. In contrast to the results of continuous decidualization for 4 days (Muter et al. 2015), these core clock genes did not exhibit obvious rhythmic activity in undifferentiated

Table 2 Information on the antibodies used in this study.

\begin{tabular}{ll}
\hline Antibody & \\
\cline { 1 - 2 } PER1 & Abcom \\
PRL & R\&D \\
IGFBP1 & Abcom \\
FOXO1 & CST \\
PR & CST \\
Akt & CST \\
p-Akt (Ser473) & CST \\
GAPDH & Abmart \\
Vimentin & Epitomics \\
Cytokeratin & DAKO \\
\hline
\end{tabular}

\begin{tabular}{l}
\hline Catalog \\
\hline Ab136451 \\
AF682 \\
Ab181141 \\
2880 \\
8757 \\
46915 \\
4060 S \\
P30008 \\
$2707-1$ \\
Z0622
\end{tabular}

\begin{tabular}{lll}
\hline \multicolumn{2}{c}{ Applications } \\
\hline WB & \multicolumn{1}{c}{$\mathrm{IF}$} \\
\cline { 1 - 1 } $1: 500$ & & $1: 400$ \\
$1: 10$ & & $/$ \\
$1: 1000$ & & $/$ \\
$1: 1000$ & & $1: 200$ \\
$1: 500$ & $/$ \\
$1: 1000$ & & $/$ \\
$1: 1000$ & & $/$ \\
$1: 2000$ & & $/$ \\
$/$ & $1: 200$ \\
$/$ & $1: 200$ \\
\hline
\end{tabular}

Akt, AKT serine/threonine kinase 1; FOXO1, forkhead box O1; GAPDH, glyceraldehyde-3-phosphate dehydrogenase; IGFBP1, insulin-like growth factor-binding protein 1; p-Akt, phospho AKT serine/threonine kinase 1; PER1, period 1; PR, progesterone receptor; PRL, prolactin. 
culture. Furthermore, they did not significantly flatten in decidualized culture, indicating that the rhythm of core circadian genes may be unnecessary for the proliferationdifferentiation transition in early decidualization. Nonetheless, we noticed that no statistically significant differences in every time point of these core clock genes between undifferentiated state and decidualized state, except for the elevated expression of PER1 mRNA at 4, 8, 28 and $32 \mathrm{~h}$ (Fig. 1A), showing that PER1 seems to be involved in the initiation of decidualization.

Using an in vitro decidualization system, the mRNA and protein levels of prolactin (PRL) and insulin-like growth factor-binding protein-1 (IGFBP1), two reliable decidual markers, increased steadily in decidualized immortalized HESCs for 2, 4, 6, or 8 days, accounting for the robustness of the experimental system (Supplementary Fig. 1A, B, C, $\mathrm{D}$ and $\mathrm{E}$, see section on supplementary data given at the end of this article). We profiled the expression of PER1 in this scheme and observed that the PER1 transcript level was substantially augmented in decidualized cells (Fig. 1B). Western blot analysis also confirmed the dramatic elevations in PER1 of up to three-fold over a 6-day period (Fig. 1C and D). Immunofluorescence staining showed that PER1 protein was weakly expressed in the cytoplasm and nuclei of stromal cells; however, it was predominantly expressed in the nuclei of stromal cells after differentiation for 4 days, consistent with the patterns in decidua cells in early pregnancy in vivo (Fig. 1E). These data show that the upregulation of PER1, rather than other clock genes, is a striking feature of decidualization and may also be involved in the maintenance of decidualization.

Furthermore, we measured PER1 expression levels in human endometrial biopsy samples from each phase of the menstrual cycle and found that PER1 mRNA expression increased significantly in the mid-secretory phase of the cycle (Fig. 1F). Next, we compared the PER1 expression of mid-luteal endometrial biopsies obtained from nine women with infertility diagnosed with EMS, a chronic syndrome characterized by infertility due to $\mathrm{P}_{4}$ resistance and disrupted decidualization (Wetendorf \& Demayo 2014), and ten women with normal fertility. The endometrial PER1 mRNA expression in EMS patients was significantly lower than that in control patients (Fig. 1G), indicating that PER1 may be related to $\mathrm{P}_{4}$ responsiveness.

\section{PER1 expression is regulated by progesterone as early as the onset of decidualization}

To assess the impacts of $\mathrm{P}_{4}$ and cAMP signaling on PER1 expression on the onset of decidualization, we first treated immortalized HESCs with db-cAMP and MPA separately or in combination for $6 \mathrm{~h}$. The increase in PER1 expression was more pronounced with MPA than with db-cAMP and combined treatment resulted in an additive effect (Fig. 2A). Next, we examined whether RU486 (a PR inhibitor) could diminish $\mathrm{P}_{4}$-activated PER1 expression. The PER1 mRNA levels were significantly lower in RU486-treated cells than in control cells exposed to MPA alone or to a combination of db-cAMP and MPA (Fig. 2B and C), indicating that the expression of PER1 mRNA is regulated mainly by $\mathrm{P}_{4}$-PR signaling from the beginning of decidualization.

Considering that RU486 is also an antagonist of the glucocorticoid receptor, which is expressed in uterine stromal cells (Whirledge et al. 2015) and that glucocorticoids can increase PER1 levels in a variety of cell types (Reddy et al. 2012, Cheon et al. 2013), an immortalized PGR-knockout (PGR-KO) HESC line was constructed with CRISPR/Cas9 technology to confirm this potential regulation. The Sanger sequencing results confirmed that 16 nucleic acids were inserted into and that 170 nucleic acids were deleted from coding sequence of one allele, while six nucleic acids were inserted into and 160 nucleic acids were deleted from the coding sequence of the other allele; these changes resulted in frameshift mutations and mutants with predicted deletions of 333 and 303 amino acids, respectively (Fig. 2D). Decidualized control (empty vector) and PGR-KO cell lines were analyzed by qRT-PCR and Western blot, and the results showed decreased mRNA expression of PRL and IGFBP1 and a loss of PRA/PRB and PER1 protein in PGR-KO cells (Fig. 2E and Supplementary Fig. 2A and B). Induction by MPA for $6 \mathrm{~h}$ attenuated the increase in the PER1 mRNA level in the MPA-treated group compared to the control group (Fig. 2F).

\section{PER1 is related to $\mathrm{P}_{4}$ signal responsiveness}

To ascertain whether PER1 is regulated by PR and PER1 is related with $\mathrm{P}_{4}$-PR signaling responsiveness in decidualization, a PER1-knockout (PER1-KO) immortalized HESC line was constructed using the CRISPR/Cas9 system. The Sanger sequencing results confirmed that two nucleic acids were deleted from the coding sequence of one allele and that 40 nucleic acids were deleted from the coding sequence of the other allele, resulting in the predicted proteins being shortened to 210 and 213 amino acids, respectively (Fig. 3A). Control (empty vector) and PER1-KO cell lines were analyzed by Western blot analysis, and loss of the PER1 protein 
A
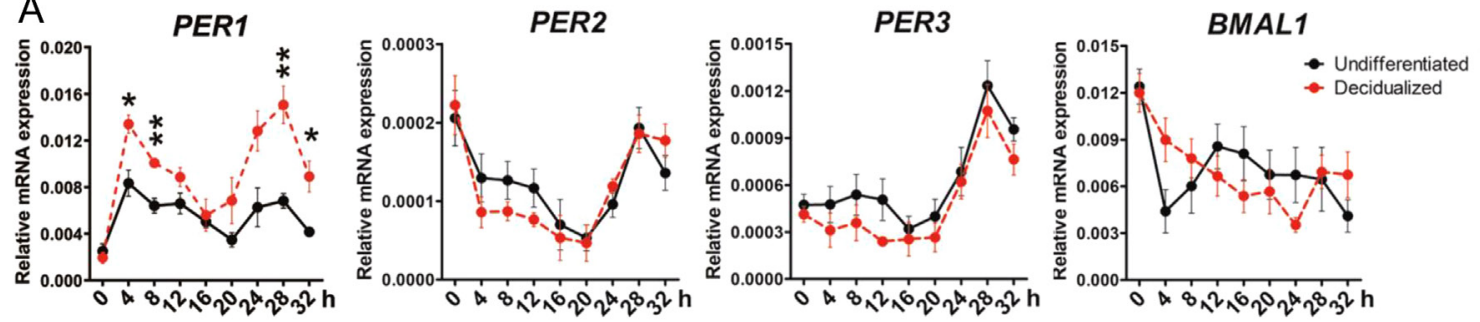

B
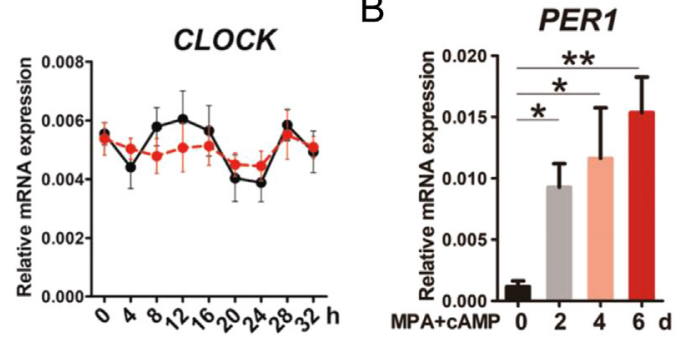

C

D

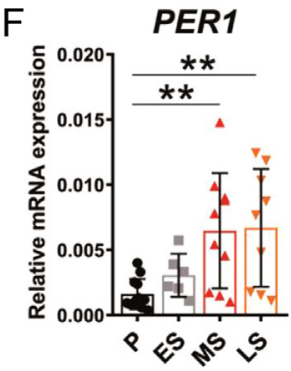

E
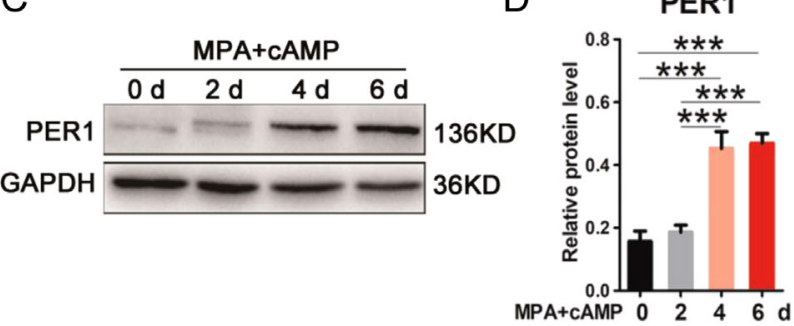

MPA+CAMP
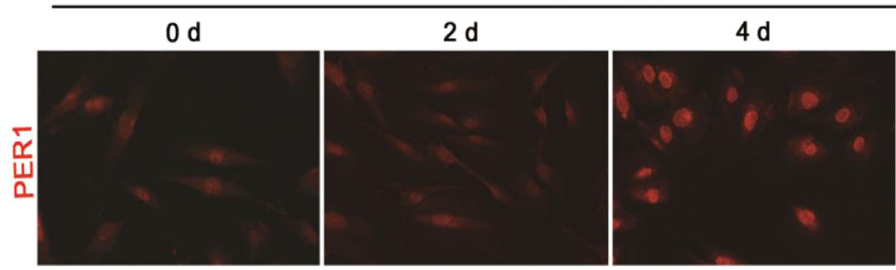
$4 \mathrm{~d}$ (negative control)
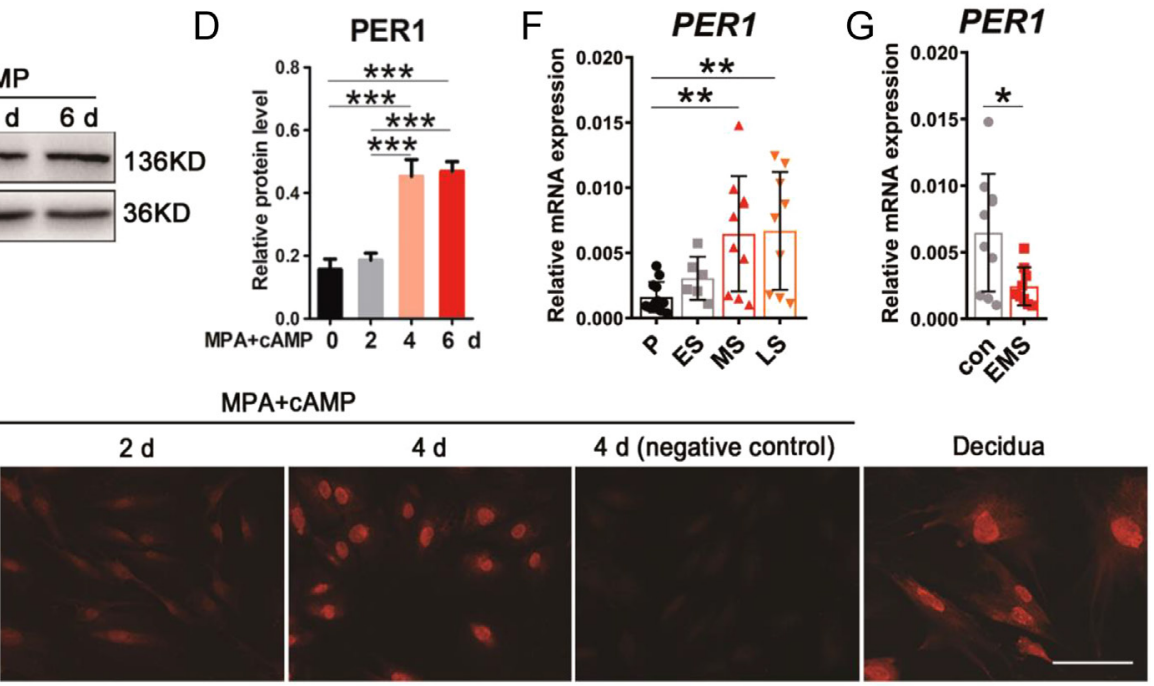

\section{Figure 1}

The expression of the circadian gene PER 1 increased immediately and remained elevated during decidualization. (A) Immortalized HESCS were first treated with dexamethasone for $30 \mathrm{~min}$ and then with estradiol (undifferentiated cells) or db-cAMP plus MPA (decidualized cells) for $32 \mathrm{~h}$, and the transcript levels of seven core clock genes were measured at the indicated time points ( $n=3$ per independent experiment). The values are normalized to the GAPDH expression level. Analysis was performed between the undifferentiated group and the decidualized group using two-way repeated measures ANOVA with Bonferroni's post hoc test. $* P<0.05, * * P<0.01$. ( $B, C$ and $D$ ) The mRNA and protein levels of PER1 in immortalized HESCs after treatment with MPA plus db-cAMP for $0,2,4$ or 6 days ( $n=4$ per independent experiment). The values are normalized to the GAPDH expression level. Analysis was performed with one-way ANOVA and Bonferroni's post hoc test. $* P<0.05, * * P<0.01, * \star * P<0.001$. (E) Immunofluorescence staining of PER1 in immortalized HESCs after treatment with MPA plus db-CAMP for 0,2 , and 4 days. Human decidual cells cultured in vitro during early pregnancy were used as positive control. The sample without primary antibody was served as a negative control. Scale bars, $100 \mu \mathrm{M}$. (F) qRT-PCR analysis of PER1 mRNA levels in endometria from the proliferative ( $n=12$ samples), early secretory ( $n=6$ samples), mid-secretory $(n=10$ samples), and late secretory ( $n=9$ samples) stages of the menstrual cycle. The values are normalized to the GAPDH expression level. Analysis was performed with one-way ANOVA and Bonferroni's post hoc test. ES, early secretory phase; $M S$, mid-secretory phase; $L S$, late secretory phase; $P$, proliferative phase. ${ }^{*} P<0.01$. (G) Endometrial $P E R 1$ transcript levels during the mid-secretory phase in fertile women ( $n=10$ samples) versus infertility patients with endometriosis (EMS) ( $n=9$ samples). The values are normalized to the GAPDH expression level. Analysis was performed with Student's unpaired two-tailed $t$-test. $* P<0.05$.

was observed in PER1-KO cells (Fig. 3B). We found that PGR mRNA levels were not affected in PER1-KO cells after combined db-cAMP and MPA treatment (Fig. 3C), but the expression levels of the DKK1 and HTBS1, two PR downstream genes have been identified (Mazur et al. 2015), were significantly decreased (Fig. 3D and E). These results suggest that PER1 may be associated with decreased $\mathrm{P}_{4}$-PR signaling responsiveness. 
A

D human $P G R$

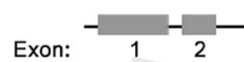

B

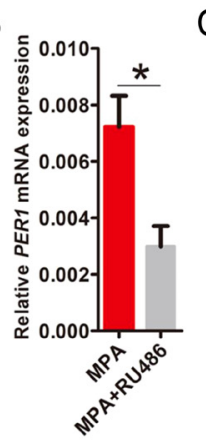

$C_{\text {. }}$

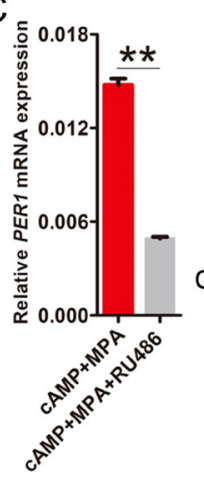

E
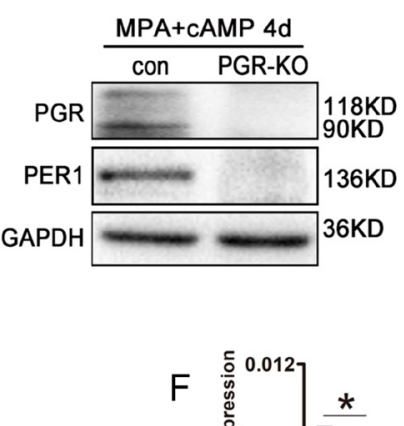

\section{$\mathrm{F}$}

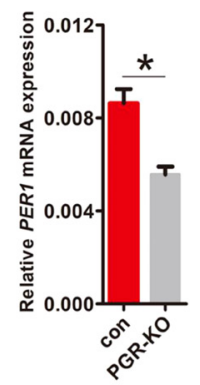

Figure 2

PER1 expression is regulated by progesterone as early as the onset of decidualization. (A) PER1 mRNA levels in immortalized HESCs at $6 \mathrm{~h}$ after treatment with MPA and db-cAMP separately or in combination ( $n=3$ per independent experiment). The values are normalized to the GAPDH expression level. Analysis was performed with one-way ANOVA and Bonferroni's post hoc test. $* \star P<0.01, * \star \star P<0.001$. (B) $P E R 1$ mRNA levels in immortalized HESCs at $6 \mathrm{~h}$ after treatment with MPA with or without RU486 ( $n=5$ per independent experiment). The values are normalized to the GAPDH expression level. Analysis was performed with Student's unpaired two-tailed $t$-test. $* P<0.05$. (C) $P E R 1$ mRNA levels in immortalized HESCs at $6 \mathrm{~h}$ after treatment in combination of MPA and db-cAMP with or without RU486 ( $n=3$ per independent experiment). The values are normalized to the GAPDH expression level. Analysis was performed with Student's unpaired two-tailed $t$-test. $* * P<0.01$. (D) Schematic diagram of the PGR knockout cell line DNA sequence by Sanger sequencing. (E) Control (empty vector) and PGR-knockout (PGR-KO) cell lines were analyzed by Western blotting after treatment with MPA plus db-CAMP for 4 days. (F) PER1 mRNA levels of control and PGR-KO HESCs after treatment with MPA at $6 \mathrm{~h}$ ( $n=5$ per independent experiment). The values are normalized to the GAPDH expression level. Analysis was performed with Student's unpaired two-tailed $t$-test. * $P<0.05$. A full colour version of this figure is available at https://doi.org/10.1530/JOE-19-0284.

\section{Progesterone receptor modulates PER1 via direct transcriptional activation}

A previous study revealed Per1 as a direct transcriptional target of PR in the mouse uterus (Rubel et al. 2012); we hypothesized that this regulatory mechanism also exists in the human endometrium. Using two reported progesterone response elements (PREs), 5'-ACNNNNNGT-3' (Lambert et al. 2018) and 5'-TGTNCN-3' (Ballare et al. 2013), we found four putative PRE-binding sites located in the PER1 promoter (Fig. 4A and B). After cells were allowed to decidualize for $24 \mathrm{~h}$, chromatin immunoprecipitation (ChIP) analysis using a PR antibody showed that decidualization was associated with conspicuous binding
A

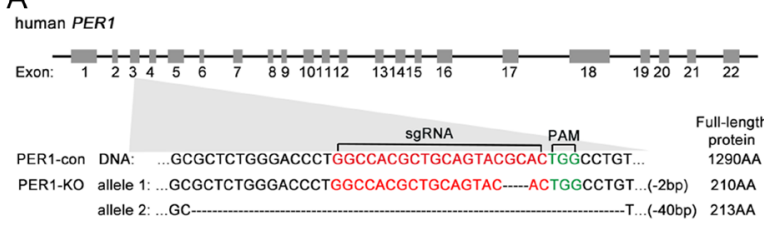

C

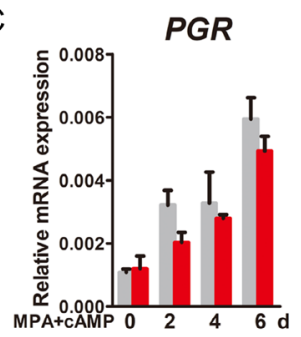

B
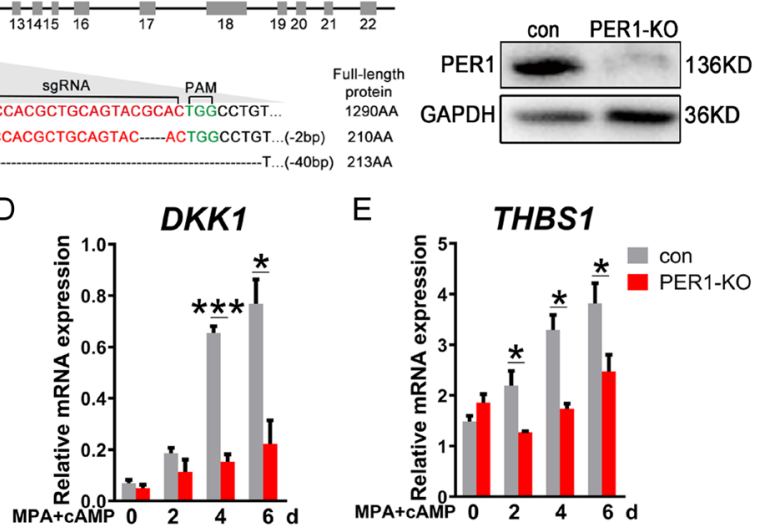

$\mathrm{E}$

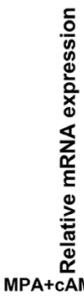

THBS1

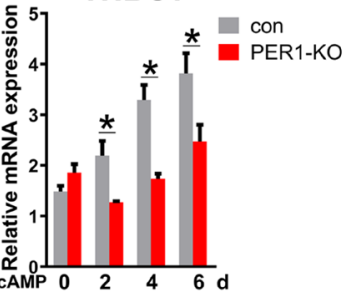

\section{Figure 3}

PER1 is related to $\mathrm{P}_{4}$ signal responsiveness. (A) Schematic diagram of the PER1-knockout (PER1-KO) cell line DNA sequence by Sanger sequencing. (B) Control (empty vector) and PER1-KO cell lines were analyzed by Western blotting. (C, D and E) PGR, DKK1 and HTBS1 mRNA levels in control and PER1-KO HESCS after treatment with MPA plus db-cAMP at 0, 2, 4, and 6 days ( $n=3$ per independent experiment). The values are normalized to the GAPDH expression level. Analysis was performed between the control group and the PER1-KO group using two-way repeated-measures ANOVA with Bonferroni's post hoc test. $* P<0.05, * * * P<0.001$. A full colour version of this figure is available at https://doi.org/10.1530/JOE-19-0284. 
A
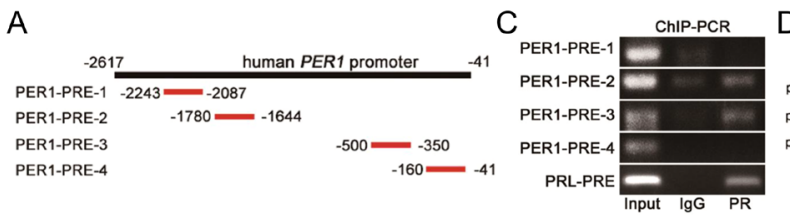

\section{PGL3-Basic}

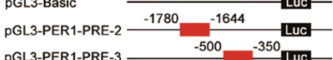

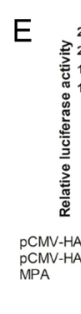

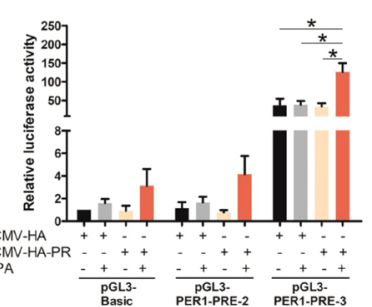

\section{Figure 4}

The progesterone receptor modulates PER1 via transcriptional activation. (A and B) Schematic diagram and sequence of the putative progesterone response element (PRE) on the PER1 promoter. (C) Chromatin immunoprecipitationPCR (ChIP-PCR) analysis of immortalized HESCS after treatment with MPA plus db-cAMP for $24 \mathrm{~h}$. The input:IgG:PR ratio was 1:12:12. (D) Schematic diagram of the PGL3-PER1-PRE-2 and PGL3-PER1PRE-3 reporter constructs for the promoter assay. (E) Luciferase assays of the cis-activation potential of the region containing pGL3-PER1-PRE-2 and PGL3-PER1-PRE-3 in the presence of control plasmid, PR overexpression plasmid or MPA $(n=3$ per independent experiment). Analysis was performed from intra-group comparison of pGL3-Basic, pGL3-PER1-PRE-2 and pGL3-PER1PRE-3 with one-way ANOVA and Bonferroni's post hoc test. $* P<0.05$. A full colour version of this figure is available at https://doi.org/10.1530/ JOE-19-0284. of PR at the locus PER1-PRE-2 (bp -1780 to -1644) and PER1-PRE-3 (bp -500 to -350) (Fig. 4C). These two sequences were inserted into luciferase reporter vectors (Fig. 4D), and we found that the activity of the reporter with the PER1-PRE-3 insert was significantly higher than that of both the PER1-PRE-2 reporter and the control reporter; in addition, the reporter with PER1-PRE-3 was actively induced by $\mathrm{P}_{4}$-PR signaling (Fig. 4E). These results show that the expression of PER1 is potentially regulated by direct binding of PR to the PER1-PRE-3 sequence.

\section{PER1 deficiency leads to impaired HESC decidualization via decreased FOX01 protein levels}

To investigate the function of PER1 in decidualization, siRNA-mediated knockdown was performed on primary HESCs. Successful establishment of primary stromal cell cultures was confirmed by positive staining for vimentin and negative staining for cytokeratin (Supplementary Fig. 2C). The qRT-PCR and Western blot analysis results confirmed that PER1 mRNA and protein levels were significantly lower (Fig. 5A and B) and that decidualized cells were also significantly smaller in the knockdown group than in the control group (Fig. 5C and D). These changes were accompanied by severely compromised induction of IGFBP1 and PRL at the mRNA levels (Fig. 5E and F).

Since critical decidual markers such as Foxo1, Wnt4 and Stat3 have been reported to be regulated transcriptionally by Per1 in the mouse liver (Koike et al. 2012), we assessed the mRNA and protein levels of these molecules after PER1 knockdown. Intriguingly, PER1 knockdown in primary HESCs exerted no significant impact on the transcripts of these genes (data not shown) and inhibited only FOXO1 protein expression (Fig. 6A, $\mathrm{B}$ and $\mathrm{C})$. Furthermore, the immunofluorescence staining results showed that FOXO1 was strongly expressed in the nuclei and cytoplasm of decidual cells in the control group after 6 days of decidualization, but distinctly decreased in both the nuclei and cytoplasm of cells in the siPER1 group (Fig. 6D). The declines in the levels of the FOXO1, PRL, and IGFBP1 proteins were also observed in PER1-KO cells (Supplementary Fig. 2D, E, F and G), consistent with the results in knockdown cells. In primary HESCs with PER1 knockdown and FOXO1 overexpression, the aberrant decidualization was attenuated, and IGFBP1 expression levels were rescued (Fig. 6E, F and G), manifesting that PER1 promotes decidualization by regulating FOXO1.

\section{PER1 regulates FOX01 protein stability through the calpain pathway}

To demonstrate whether the existence of another regulatory mechanism underlying the decreases of FOXO1 protein levels in the absence of PER1, as shown in Fig. 7A, B and C, the protein levels of Akt and phosphorylated Akt, the upstream regulator of FOXO1 was estimated and in siCON and siPER1 primary decidualizing cells. The comparable level of p-AKT in both groups indicated that the mRNA or protein stability of FOXO1 could be regulated by PER1 deficiency.

The half-life of FOXO1 protein was measured after inhibition of total protein synthesis. When control and 
A

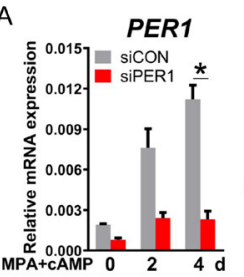

B

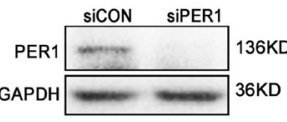

C

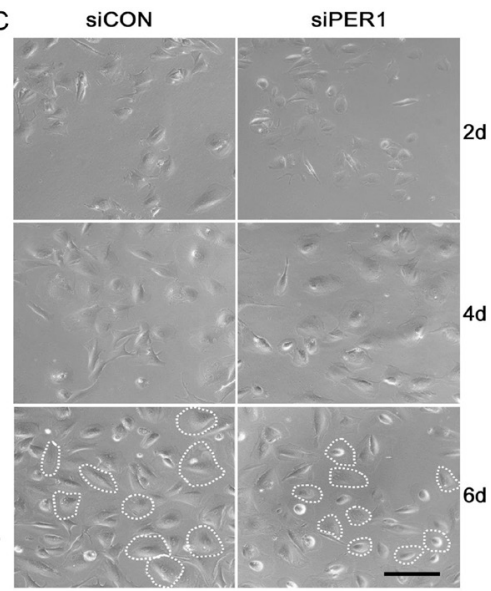

\section{Figure 5}

PER1 is indispensable for HESC decidualization. (A) PER1 mRNA levels in primary HESCs after transfection with siRNAs for $24 \mathrm{~h}$ followed by treatment with MPA plus db-cAMP at 0,2 and 4 days ( $n=3$ per independent experiment). The values are normalized to the GAPDH expression level. Analysis was performed between the control group (siCON) and the siPER1 group using two-way repeated-measures ANOVA with Bonferroni's post hoc test. * $P<0.05$. (B) PER1 protein levels in primary HESCs after transfection with siRNAs for $24 \mathrm{~h}$. (C) Morphology of primary HESCs after transfection with siRNAs for $24 \mathrm{~h}$ followed by treatment with MPA plus db-cAMP at 2, 4 and 6 days. The size of a single decidual cell is outlined with a white dotted line. Scale bars, $100 \mu \mathrm{M}$. (D) The cell size of primary HESCs after transfection with siRNAs for $24 \mathrm{~h}$ followed by treatment with MPA plus db-cAMP for 6 days ( $n=50$ cells). Analysis was performed with Student's unpaired two-tailed $t$-test. ${ }^{*} P<0.05$. (E and F) PRL and IGFBP1 mRNA levels in primary HESCS after transfection with siRNAs for $24 \mathrm{~h}$ followed by treatment with MPA plus db-cAMP at 0, 2 and 4 days ( $n=3$ per independent experiment). The values are normalized to the GAPDH expression level. Analysis was performed between the control group and the siPER1 group using two-way repeated measures ANOVA with Bonferroni's post hoc test. ${ }^{*} P<0.05, * \star P<0.01$. A full colour version of this figure is available at https://doi.org/10.1530/JOE-19-0284

PER1-KO HESCs were treated with CHX for 0, 1, 2, or $4 \mathrm{~h}$ after decidualization for 2 days, the most remarkable observation was that the half-life of FOXO1 protein was $1 \mathrm{~h}$ shorter in PER1-KO cells than in control cells (Fig. 7D), suggesting that PER1 knockout affects FOXO1 protein stability. Treatment of cells with the calpain inhibitor MG101, the proteasome inhibitor MG132, the autophagy inhibitor 3-MA, or all three compounds revealed that MG101 could efficiently attenuate the rapid degradation of FOXO1 protein in PER1-KO cells, while the combination of the three compounds had no synergistic effect (Fig. 7E). These findings indicate that PER1 regulates FOXO1 protein stability mainly through the calpain-mediated degradation pathway.

\section{Discussion}

Female reproduction in humans is associated with a monthly cyclic process. With follicle growth and ovulation each month, the dynamic secretion of estradiol and $\mathrm{P}_{4}$ plays a dominant role in orchestrating changes in uterine epithelial cells and stromal cells that prepare the endometrium for a successful pregnancy. It was first reported in 2002 that circadian genes are expressed in the mouse uterus (Johnson et al. 2002). $\mathrm{P}_{4}$ treatment can induce high expression of Per1 in the epithelium and stroma of the rat uterus (He et al. 2007). In addition, the clock protein PER1 is upregulated along with decidual transformation of HESCs (Muter et al. 2015). Likewise, our results proved that the expression of PER1 increased immediately and remained elevated during decidualization in vivo and in vitro due to transcriptional activation by $\mathrm{PR}$, suggesting that PER1 is the major response factor for $\mathrm{P}_{4}$-PR signaling. Per1-mutant female mice show normal numbers of implantation sites but have reduced litter sizes (Pilorz \& Steinlechner 2008), confirming that Per1 deficiency may lead to unsustainable pregnancy. Decidualization provides an immune-privileged microenvironment that enables embryo implantation as well as abundant nutrients that facilitate early embryonic development, thereby maintaining gestation (Zhang et al. 2013), the compromised decidualization might thus be related to postimplantation loss resulting from the Per1 mutation. In agreement with this idea, we found that PER1 levels were decreased in the eutopic endometrium of patients with EMS, which is relevant to the finding that $\mathrm{P}_{4}$ resistance causes decreased fecundity.

In addition to the progesterone signaling pathway, which is activated by $\mathrm{P}_{4}$ produced by the corpus luteum, the cAMP signal transduction cascade is activated by local molecules of the endometrium in the initiation 
A

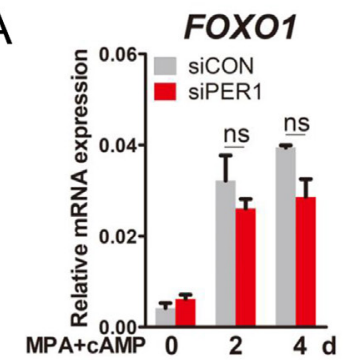

B

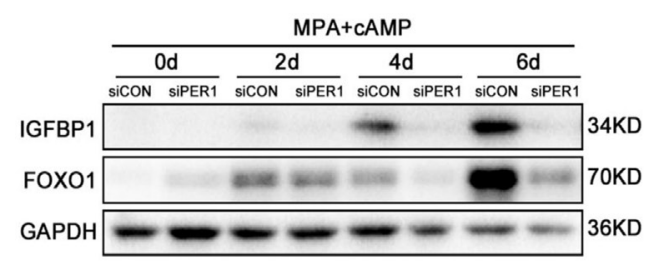

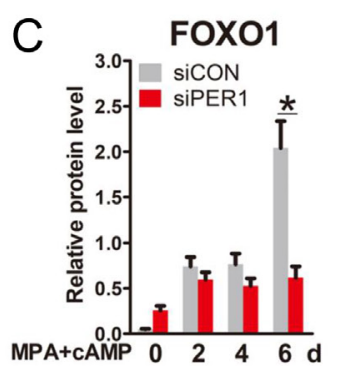

E
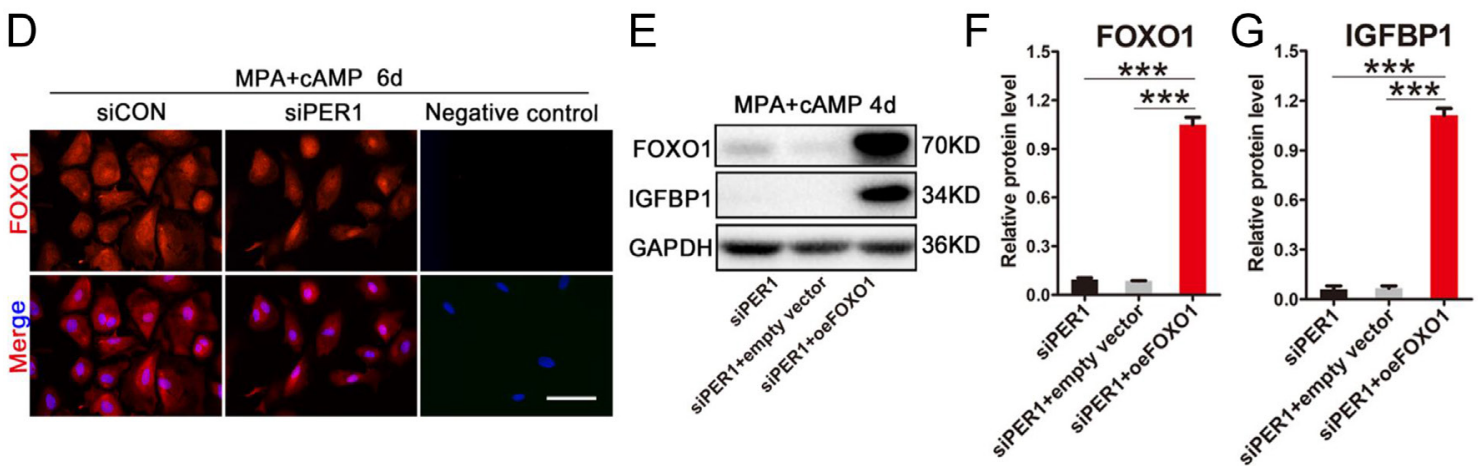

\section{Figure 6}

PER1 deficiency leads to decreased FOXO1 protein levels. (A, B and C) FOXO1 mRNA and protein levels in primary HESCs after transfection with siRNAs for $24 \mathrm{~h}$ followed by treatment with MPA plus db-cAMP at 0, 2, 4 and 6 days ( $n=3$ per independent experiment). The values are normalized to the GAPDH expression level. Analysis was performed between the control group (siCON) and the siPER1 group using two-way repeated-measures ANOVA with Bonferroni's post hoc test. ${ }^{*} P<0.05$. (D) FOXO1 immunofluorescence staining of primary HESCs after transfection using siRNAs for $24 \mathrm{~h}$ followed by treatment with MPA plus db-cAMP for 6 days. The primary HESC after treatment with MPA plus db-cAMP for 6 days without incubation by primary antibody was served as a negative control. Scale bars, $100 \mu \mathrm{M}$. (E, F and G) Primary HESCs were infected with a FOXO1-overexpressing virus (oeFOXO1) for $24 \mathrm{~h}$, and then transfected with siPER1 for $24 \mathrm{~h}$ and next treated with MPA plus db-cAMP for 4 days ( $n=3$ per independent experiment). The FOXO1 and IGFBP1 protein levels were detected by Western blotting. The values are normalized to the GAPDH expression level. Analysis was performed with one-way ANOVA and Bonferroni's post hoc test. $* \star \star P P<0.001$.

of decidualization, sensitizing endometrial cells to the actions of progesterone (Gellersen \& Brosens 2003). $\mathrm{P}_{4}$ is only a weak inducer of the decidual phenotype unless combined with cAMP, but it plays a critical role in maintaining the decidual phenotype (de Ziegler et al. 1998). The mRNA levels of PER1, a direct target gene of $P R$, increased rapidly when decidualization was induced in vitro for $4 \mathrm{~h}$, suggesting the possibility that $\mathrm{P}_{4}$ signaling is also involved in the initiation of decidualization. $\mathrm{P}_{4}$ has long been known to exhibit circadian secretion, similar to other hormones (Kottler et al. 1989, Fujimoto et al. 1990); yet, the expression of its direct target gene PER1 is arrhythmic, suggesting that $\mathrm{P}_{4}$ is probably not a zeitgeber.

In mammals, the integrated endocrine system controls and adjusts physiological processes by secreting hormones. Previous findings have suggested that the endocrine system could serve as a major clock output (Lamia et al. 2011), and some rhythmic hormones can also activate the circadian system to regulate the expression of genes critical for physiological mechanisms (McNamara et al. 2001).
We observed sustained activation of the clock gene PER1 in response to $\mathrm{P}_{4}$ signaling during the decidualization of HESCs. In fact, upregulation of PER1 is a generic feature in many tissues in response to certain types of hormonal stress (Mansuy et al. 2009, Mendoza et al. 2011, Smarr et al. 2013, Bohacek et al. 2015). This expression pattern indicates that PER1 might be the most sensitive and susceptible effector in the clock system. Previous research has shown that both transcriptional and posttranscriptional regulation underlie the activity of clock system components, including PER1, and that posttranscriptional mechanisms account for more than half of the regulation (Koike et al. 2012), suggesting that transcriptional regulation is not the main mode of operation of the clock system. Our observation that PER1 deficiency impairs the decidual transformation of the human endometrium through posttranslational regulation of FOXO1, a terminal differentiation-related gene that is evolutionarily conserved in eutherian animals and has acquired the ability to respond to deciduogenic signals (Park et al. 2016), strongly reinforces the idea that a 
A

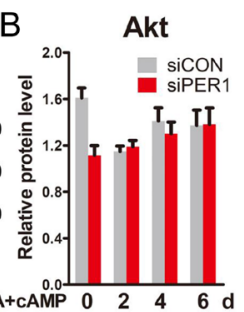

C p-Ser473 Akt

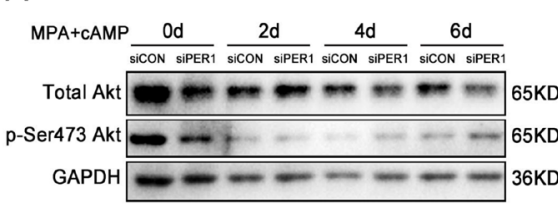

B

D

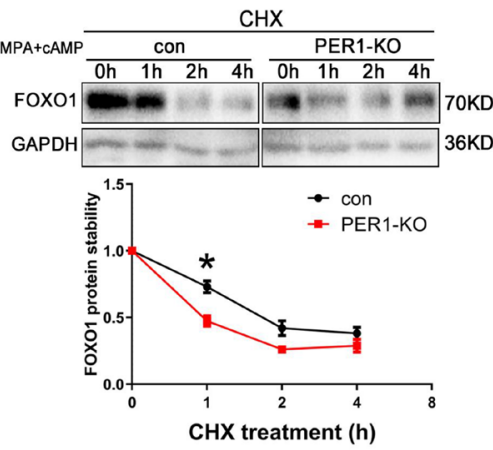

E

E MPA+CAMP PER1-KO
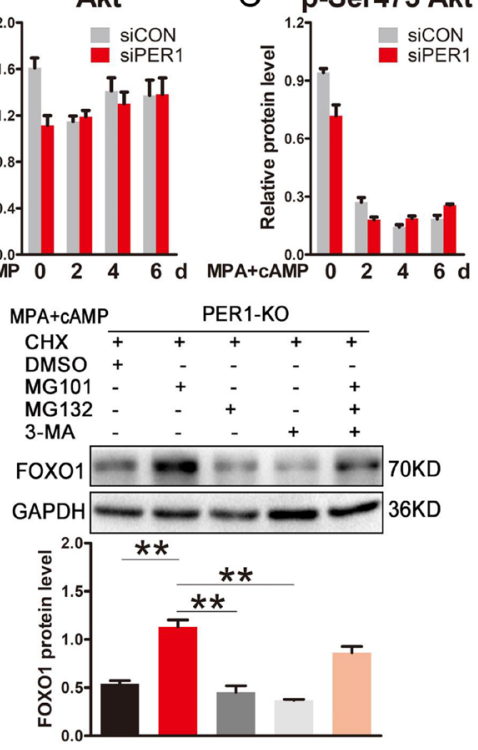

Figure 7

PER1 regulation of FOXO1 protein stability through the calpain pathway. (A, B and C) Akt and phosphor-Akt (Ser473) protein levels in primary HESCs after transfection with siRNAs for $24 \mathrm{~h}$ followed by treatment with MPA plus db-CAMP at $0,2,4$ and 6 days ( $n=3$ per independent experiment). The values are normalized to the GAPDH expression level. Analysis was performed between the control group (siCON) and the siPER1 group using two-way repeatedmeasures ANOVA with Bonferroni's post hoc test. No statistically significant differences were detected. (D) After treatment with MPA plus db-cAMP for 2 days, immortalized HESCs were treated with $\mathrm{CHX}$ for $0,1,2$ and $4 \mathrm{~h}$, and the FOXO1 levels in the control (empty vector) and PER1-KO cells were detected by Western blotting ( $n=3$ per independent experiment). The values are normalized to the GAPDH expression level. Analysis was performed between the control group and the PER1-KO group using two-way repeated-measures ANOVA with Bonferroni's post hoc test. * $P<0.05$. (E) After treatment with MPA plus db-cAMP for 2 days, immortalized HESCs were treated with CHX for $2 \mathrm{~h}$ along with DMSO, MG101, MG132 or 3-MA. The FOXO1 levels in the control and PER1-KO cells were detected by Western blotting ( $n=3$ per independent experiment). The values are normalized to the GAPDH expression level. Analysis was performed with one-way ANOVA and Bonferroni's post hoc test. $* \star P<0.01$. A full colour version of this figure is available at https://doi. org/10.1530/JOE-19-0284.

posttranslational mechanism is involved in the circadian gene regulatory network.

The circadian rhythm is a precisely tuned process. In the human uterus, downregulation of PER2 in the endometrium marks the transition from oscillatory to nonoscillatory stroma, synchronizing the preimplantation embryo and surrounding decidual cells for fetal-maternal interactions (Muter et al. 2015). This mechanism provides a likely explanation for the abolished rhythms in differentiating HESCs. However, cell differentiation results in loss of rhythm in the clock systems of the testis and thymus, which are composed primarily of differentiating cells and exhibit constant rather than cyclic expression of circadian clock genes (Alvarez et al. 2003, Morse et al. 2003). PER2 is necessary to maintain the undifferentiated state of CD34 (+) cells, while decreased PER2 and loss of rhythm are associated with diabetes-induced propensity toward myeloid differentiation (Bhatwadekar et al. 2015). Per3 has a prominent rhythm in mouse adipocyte precursor cells and restrains adipogenesis, a process of cell differentiation, by directly regulating Klf15 expression
(Aggarwal et al. 2017). Similarly, endometrial stromal decidualization is also a cell differentiation process in which fusiform stromal cells in a proliferative state differentiate into cobblestone-shaped specialist decidual cells to implement embryo selection and promote embryo implantation; this process represents another promising yet disputed explanation of how the abolition of the PER1 rhythm is related to cell differentiation.

In conclusion, our study introduces a mechanism of clock reaction to $\mathrm{P}_{4}$ signaling that allows endometrial stromal cells to transform into decidual cells. We show that a phenomenon associated with $\mathrm{P}_{4}$-PR signaling triggers the uterine stromal cell proliferation-differentiation transition apart from maintaining this process. Our findings are likely relevant to compromised decidual responses that predispose women to reproductive failure.

\section{Supplementary data}

This is linked to the online version of the paper at https://doi.org/10.1530/ JOE-19-0284. (c) 2019 Society for Endocrinology Published by Bioscientifica Ltd. Printed in Great Britain 


\section{Declaration of interest}

The authors declare that there is no conflict of interest that could be perceived as prejudicing the impartiality of the research reported.

\section{Funding}

This work was supported by the National Basic Research Program of China (grant number 2017YFC1001402, H Wang), the National Natural Science Foundation of China (grant number 81771543, YZ Zhang; grant numbers 81830045 and 81490744, H Wang), Fundamental Research Funds for the Central Universities (grant number 2072018003, S Kong), and Excellent Youth Foundation of Hubei Province Department of Education (grant number Q20122402, Y Zhang).

\section{Author contribution statement}

$\mathrm{Y}$ Zhang, $\mathrm{Y}$ Z Zhang, S Kong and $\mathrm{H}$ Wang conceived and designed the research; $Y$ Zhang, N Meng, Y Jiang, N Yang, K Wu and J Wu performed the research; $Y$ Zhang analyzed the results; and $Y$ Zhang, $\mathrm{H}$. Bao, $Y Z Z$ Zhang, and $S$ Kong wrote the manuscript. All authors reviewed the manuscript.

\section{References}

Aggarwal A, Costa MJ, Rivero-Gutierrez B, Ji L, Morgan SL \& Feldman BJ 2017 The circadian clock regulates adipogenesis by a Per3 crosstalk pathway to Klf15. Cell Reports 21 2367-2375. (https://doi. org/10.1016/j.celrep.2017.11.004)

Alvarez JD, Chen D, Storer E \& Sehgal A 2003 Non-cyclic and developmental stage-specific expression of circadian clock proteins during murine spermatogenesis. Biology of Reproduction 69 81-91. (https://doi.org/10.1095/biolreprod.102.011833)

Ballare C, Castellano G, Gaveglia L, Althammer S, Gonzalez-Vallinas J, Eyras E, Le Dily F, Zaurin R, Soronellas D, Vicent GP, et al. 2013 Nucleosome-driven transcription factor binding and gene regulation. Molecular Cell 49 67-79. (https://doi.org/10.1016/j. molcel.2012.10.019)

Bhatwadekar AD, Yan Y, Stepps V, Hazra S, Korah M, Bartelmez S, Chaqour B \& Grant MB 2015 miR-92a corrects CD34+ cell dysfunction in diabetes by modulating core circadian genes involved in progenitor differentiation. Diabetes 64 4226-4237. (https://doi. org/10.2337/db15-0521)

Bohacek J, Manuella F, Roszkowski M \& Mansuy IM 2015 Hippocampal gene expression induced by cold swim stress depends on sex and handling. Psychoneuroendocrinology 52 1-12. (https://doi. org/10.1016/j.psyneuen.2014.10.026)

Cheon S, Park N, Cho S \& Kim K 2013 Glucocorticoid-mediated Period2 induction delays the phase of circadian rhythm. Nucleic Acids Research 41 6161-6174. (https://doi.org/10.1093/nar/gkt307)

de Ziegler D, Fanchin R, de Moustier B \& Bulletti C 1998 The hormonal control of endometrial receptivity: estrogen (E2) and progesterone. Journal of Reproductive Immunology 39 149-166. (https://doi. org/10.1016/S0165-0378(98)00019-9)

Fujimoto VY, Clifton DK, Cohen NL \& Soules MR 1990 Variability of serum prolactin and progesterone levels in normal women: the relevance of single hormone measurements in the clinical setting. Obstetrics and Gynecology 76 71-78. (https://doi.org/10.1016/00207292(91)90375-F)

Gellersen B \& Brosens J 2003 Cyclic AMP and progesterone receptor cross-talk in human endometrium: a decidualizing affair. Journal of Endocrinology 178 357-372. (https://doi.org/10.1677/joe.0.1780357)
Gellersen B \& Brosens JJ 2014 Cyclic decidualization of the human endometrium in reproductive health and failure. Endocrine Reviews $\mathbf{3 5}$ 851-905. (https://doi.org/10.1210/er.2014-1045)

He PJ, Hirata M, Yamauchi N \& Hattori MA 2007 Up-regulation of Per1 expression by estradiol and progesterone in the rat uterus. Journal of Endocrinology 194 511-519. (https://doi.org/10.1677/JOE-07-0172)

Hirata M, He PJ, Shibuya N, Uchikawa M, Yamauchi N, Hashimoto S \& Hattori MA 2009 Progesterone, but not estradiol, synchronizes circadian oscillator in the uterus endometrial stromal cells. Molecular and Cellular Biochemistry 324 31-38. (https://doi.org/10.1007/s11010008-9981-4)

Johnson MH, Lim A, Fernando D \& Day ML 2002 Circadian clockwork genes are expressed in the reproductive tract and conceptus of the early pregnant mouse. Reproductive Biomedicine Online 4 140-145. (https://doi.org/10.1016/S1472-6483(10)61931-1)

Koike N, Yoo SH, Huang HC, Kumar V, Lee C, Kim TK \& Takahashi JS 2012 Transcriptional architecture and chromatin landscape of the core circadian clock in mammals. Science 338 349-354. (https://doi. org/10.1126/science.1226339)

Kottler ML, Coussieu C, Valensi P, Levi F \& Degrelle H 1989 Ultradian, circadian and seasonal variations of plasma progesterone and $\mathrm{LH}$ concentrations during the luteal phase. Chronobiology International 6 267-277. (https://doi.org/10.3109/07420528909056928)

Lambert SA, Jolma A, Campitelli LF, Das PK, Yin Y, Albu M, Chen X, Taipale J, Hughes TR \& Weirauch MT 2018 The human transcription factors. Cell 172 650-665. (https://doi.org/10.1016/j.cell.2018.01.029)

Lamia KA, Papp SJ, Yu RT, Barish GD, Uhlenhaut NH, Jonker JW, Downes M \& Evans RM 2011 Cryptochromes mediate rhythmic repression of the glucocorticoid receptor. Nature $\mathbf{4 8 0} 552-556$. (https://doi.org/10.1038/nature10700)

Large MJ \& DeMayo FJ 2012 The regulation of embryo implantation and endometrial decidualization by progesterone receptor signaling. Molecular and Cellular Endocrinology 358 155-165. (https://doi. org/10.1016/j.mce.2011.07.027)

Liao Y, Jiang Y, He H, Ni H, Tu Z, Zhang S, Wang B, Lou J, Quan S \& Wang H 2015 NEDD8-mediated Neddylation is required for human endometrial stromal proliferation and decidualization. Human Reproduction 30 1665-1676. (https://doi.org/10.1093/humrep/dev117)

Mansuy V, Risold PY, Glauser M, Fraichard A \& Pralong FP 2009 Expression of the GABAA receptor associated protein Gec1 is circadian and dependent upon the cellular clock machinery in GnRH secreting GnV-3 cells. Molecular and Cellular Endocrinology 307 68-76. (https:// doi.org/10.1016/j.mce.2009.02.029)

Mazur EC, Vasquez YM, Li X, Kommagani R, Jiang L, Chen R, Lanz RB, Kovanci E, Gibbons WE \& DeMayo FJ 2015 Progesterone receptor transcriptome and cistrome in decidualized human endometrial stromal cells. Endocrinology 156 2239-2253. (https://doi.org/10.1210/ en.2014-1566)

McNamara P, Seo SB, Rudic RD, Sehgal A, Chakravarti D \& FitzGerald GA 2001 Regulation of CLOCK and MOP4 by nuclear hormone receptors in the vasculature: a humoral mechanism to reset a peripheral clock. Cell 105 877-889. (https://doi.org/10.1016/s00928674(01)00401-9)

Mendoza J, Lopez-Lopez C, Revel FG, Jeanneau K, Delerue F, Prinssen E, Challet E, Moreau JL \& Grundschober C 2011 Dimorphic effects of leptin on the circadian and hypocretinergic systems of mice. Journal of Neuroendocrinology 23 28-38. (https://doi.org/10.1111/j.13652826.2010.02072.x)

Miller BH \& Takahashi JS 2013 Central circadian control of female reproductive function. Frontiers in Endocrinology 4 195. (https://doi. org/10.3389/fendo.2013.00195)

Mohawk JA, Green CB \& Takahashi JS 2012 Central and peripheral circadian clocks in mammals. Annual Review of Neuroscience $\mathbf{3 5}$ 445-462. (https://doi.org/10.1146/annurev-neuro-060909-153128)

Morse D, Cermakian N, Brancorsini S, Parvinen M \& Sassone-Corsi P 2003 No circadian rhythms in testis: Period1 expression is clock 
independent and developmentally regulated in the mouse. Molecular Endocrinology 17 141-151. (https://doi.org/10.1210/me.2002-0184)

Muter J, Lucas ES, Chan YW, Brighton PJ, Moore JD, Lacey L, Quenby S, Lam EW \& Brosens JJ 2015 The clock protein period 2 synchronizes mitotic expansion and decidual transformation of human endometrial stromal cells. FASEB Journal 29 1603-1614. (https://doi. org/10.1096/fj.14-267195)

Nakamura TJ, Moriya T, Inoue S, Shimazoe T, Watanabe S, Ebihara S \& Shinohara K 2005 Estrogen differentially regulates expression of Per1 and Per2 genes between central and peripheral clocks and between reproductive and nonreproductive tissues in female rats. Journal of Neuroscience Research 82 622-630. (https://doi.org/10.1002/ jnr.20677)

Nakamura TJ, Sellix MT, Menaker M \& Block GD 2008 Estrogen directly modulates circadian rhythms of PER2 expression in the uterus. American Journal of Physiology: Endocrinology and Metabolism 295 E1025-E1031. (https://doi.org/10.1152/ajpendo.90392.2008)

Nakamura TJ, Sellix MT, Kudo T, Nakao N, Yoshimura T, Ebihara S, Colwell CS \& Block GD 2010 Influence of the estrous cycle on clock gene expression in reproductive tissues: effects of fluctuating ovarian steroid hormone levels. Steroids 75 203-212. (https://doi. org/10.1016/j.steroids.2010.01.007)

O'Neill JS, Maywood ES, Chesham JE, Takahashi JS \& Hastings MH 2008 cAMP-dependent signaling as a core component of the mammalian circadian pacemaker. Science 320 949-953. (https://doi.org/10.1126/ science.1152506)

Papazyan R, Zhang Y \& Lazar MA 2016 Genetic and epigenomic mechanisms of mammalian circadian transcription. Nature Structural and Molecular Biology 23 1045-1052. (https://doi.org/10.1038/ nsmb.3324)

Park Y, Nnamani MC, Maziarz J \& Wagner GP 2016 Cis-regulatory evolution of forkhead box O1 (FOXO1), a terminal selector gene for decidual stromal cell identity. Molecular Biology and Evolution 33 3161-3169. (https://doi.org/10.1093/molbev/msw193).

Patel B, Elguero S, Thakore S, Dahoud W, Bedaiwy M \& Mesiano S 2015 Role of nuclear progesterone receptor isoforms in uterine pathophysiology. Human Reproduction Update 21 155-173. (https:// doi.org/10.1093/humupd/dmu056)

Pilorz V \& Steinlechner S 2008 Low reproductive success in Per1 and Per2 mutant mouse females due to accelerated ageing? Reproduction 135 559-568. (https://doi.org/10.1530/REP-07-0434)

Reddy AB, Maywood ES, Karp NA, King VM, Inoue Y, Gonzalez FJ, Lilley KS, Kyriacou CP \& Hastings MH 2007 Glucocorticoid signaling synchronizes the liver circadian transcriptome. Hepatology $\mathbf{4 5}$ 1478-1488. (https://doi.org/10.1002/hep.21571)

Reddy TE, Gertz J, Crawford GE, Garabedian MJ \& Myers RM 2012 The hypersensitive glucocorticoid response specifically regulates period 1 and expression of circadian genes. Molecular and Cellular Biology 32 3756-3767. (https://doi.org/10.1128/МСB.00062-12)

Rubel CA, Lanz RB, Kommagani R, Franco HL, Lydon JP \& DeMayo FJ 2012 Research resource: genome-wide profiling of progesterone receptor binding in the mouse uterus. Molecular Endocrinology 26 1428-1442. (https://doi.org/10.1210/me.2011-1355)

Smarr BL, Gile JJ \& de la Iglesia HO 2013 Oestrogen-independent circadian clock gene expression in the anteroventral periventricular nucleus in female rats: possible role as an integrator for circadian and ovarian signals timing the luteinising hormone surge. Journal of Neuroendocrinology 25 1273-1279. (https://doi.org/10.1111/jne.12104)

Wetendorf M \& Demayo FJ 2014 Progesterone receptor signaling in the initiation of pregnancy and preservation of a healthy uterus. International Journal of Developmental Biology 58 95-106. (https://doi. org/10.1387/ijdb.140069mw)

Whirledge SD, Oakley RH, Myers PH, Lydon JP, DeMayo F \& Cidlowski JA 2015 Uterine glucocorticoid receptors are critical for fertility in mice through control of embryo implantation and decidualization. PNAS 112 15166-15171. (https://doi.org/10.1073/pnas.1508056112)

Xin Q, Kong S, Yan J, Qiu J, He B, Zhou C, Ni Z, Bao H, Huang L, Lu J, et al. 2018 Polycomb subunit BMI1 determines uterine progesterone responsiveness essential for normal embryo implantation. Journal of Clinical Investigation 128 175-189. (https://doi.org/10.1172/JCI92862)

Yagita K \& Okamura H 2000 Forskolin induces circadian gene expression of rPer1, rPer2 and dbp in mammalian rat-1 fibroblasts. FEBS Letters 465 79-82. (https://doi.org/10.1016/s0014-5793(99)01724-x)

Zhang S, Lin H, Kong S, Wang S, Wang H, Wang H \& Armant DR 2013 Physiological and molecular determinants of embryo implantation. Molecular Aspects of Medicine 34 939-980. (https://doi.org/10.1016/j. mam.2012.12.011)

Zhang S, Kong S, Wang B, Cheng X, Chen Y, Wu W, Wang Q, Shi J, Zhang Y, Wang S, et al. 2014 Uterine Rbpj is required for embryonicuterine orientation and decidual remodeling via Notch pathwayindependent and -dependent mechanisms. Cell Research 24 925-942. (https://doi.org/10.1038/cr.2014.82)

Zmrzljak UP, Korenčič A, Košir R, Goličnik M, Sassone-Corsi P \& Rozman D 2013 Inducible cAMP early repressor regulates the Period 1 gene of the hepatic and adrenal clocks. Journal of Biological Chemistry 288 10318-10327. (https://doi.org/10.1074/jbc.M112.445692)

Received in final form 5 September 2019

Accepted 12 September 2019

Accepted Preprint published online 13 September 2019 https://joe.bioscientifica.com https://doi.org/10.1530/JOE-19-0284 (c) 2019 Society for Endocrinology Published by Bioscientifica Ltd. Printed in Great Britain 